\title{
Mining the Students' Chat Conversations in a Personalized e-Learning Environment
}

\author{
https://doi.org/10.3991/ijet.v14i23.11031 \\ Amal Al-Abri ${ }^{(凶)}$, Zuhoor Al-Khanjari, Yassine Jamoussi, Naoufel Kraiem \\ Sultan Qaboos University, Muscat, Sultanate of Oman \\ p010844@ @student.squ .edu.om
}

\begin{abstract}
Providing personalized e-learning environment is normally relying on a domain model representing the knowledge to be acquired by learners and learners' characteristics to be used in the personalization process. Therefore, constructing the domain model and understanding the characteristics of the learners are very crucial in such an environment. With the inclusion of social collaboration tools for collaborative learning activities, the generated data during conversations enrich with valuable information to be used for personalization. However, when considering chat conversations as a source for constructing the domain model, there is a need to perform a mining technique for chat conversations in order to extract the semantic relations from the user-generated contents hidden inside these conversations. As well as the learner's characteristics like learning style and knowledge level expressed during conversations. Thus in this paper, we are aiming for the best utilization of chat conversation by proposing a model containing a rule-based technique as a form of mining technique. This mining aims at extracting the semantic relations to build the domain model as an ontology-based depiction. In addition, the mining model is proposed to perform some collaborative filtering techniques to identify the learning styles and knowledge level of the learners.
\end{abstract}

Keywords - Chat conversations, Collaborative learning, Mining model, Personalized e-Learning, Ontology

\section{$1 \quad$ Introduction}

The use of collaboration tools for discussion and learning is very common nowadays. With the adoption of collaboration scenario during learning, learners enrich chat conversations with valuable data related to the domain under discussion. As well as information related to learners' characteristics such as preferences, learning style, and knowledge level. Thus, there is a need for the best utilization of this valuable data in an effective way to support the learning process. However, to extract useful information to provide personalized e-learning environment, there is a need to collect these conversations and analyze them [1]. To do so, the mining technique is required. This involves applying some important tasks to the data to be suitable for information extraction. These tasks are; the preprocessing of the collected data, the term/concept 
extraction, relationship discovery, representation of extracted concept and relations, and finally collaborative filtering task [2]. The preprocessing task is the cleaning step of the collected data to be more meaningful. The term or concept extraction and relationship discovery are the required tasks to build the domain ontology.

The domain ontology is commonly used as a source of knowledge for information retrieval from semi or unstructured text [3] like chat conversations. Ontology is a formal modeling pattern showing how different concepts are related within the same domain [4]. The semantic relationship among discussed concepts during collaboration depends on the syntactic structure of language (written text or message). Thus building the domain model requires determining the feature extraction as the language components and type of relations [4]. This process needs to ensure that the extracted concepts and relationships are semantically mapped against a domain description which normally described in the form of ontology [5]. Capturing the hidden concepts and relations within the domain requires utilizing an information extraction tool which incorporates social media contents and lexicalized grammar perceptions like GATE software. GATE has a special version/ issue for social media analysis called TwitIE. TwitIE relies on a specialized set of rules for recognizing the hashtags and user mentions in chat conversations [6]. GATE also use a set of hand-coded rules to extract the semantic relations from the text [7].

Overall, providing personalized e-learning based on constructed domain ontology considering chat discussion is a sophisticated task. Such a task requires not only building the domain ontology but also performing some collaborative filtering techniques to identify the learner's characteristics like learning style and knowledge level. These characteristics are the key parameters to provide the personalized learning package based on the grouping of learners. The current state-of-the-art of personalized e-learning supports the acquisition of domain ontology utilizing chat discussion or focuses mainly on a single parameter (knowledge level) [8], [9]. However, few existing systems can support the automatic analysis of multiple parameters using chat discussions. Besides, the utilization of generated content is limited and not fully consumed [10].

Therefore, the aim of this paper is to deliberate the best utilization of the data in chat conversation to deliver personalized e-learning. The proposed mining model in this paper aims at structuring a predefined domain ontology from chat conversations or discussions during collaborative learning activity by identifying the concepts and semantic relations exist among them. To provide the personalized package, collaborative filtering task will be performed to identify two parameters which are learning style and knowledge level.

The paper is structured as follow: Section 2 provides a background of the main concepts used in the paper. Section 3 discusses some works carried out by other researchers in the field of a mining chat conversation. Section 4 explains in details the mining model for semantic relation extraction and ontology representation including the rule-based algorithm adopted and process to reach personalization. Section 5 discusses the experiments carried out to evaluate the proposed model with a scenario about the collaboration process using the collaborative learning tool. The paper is concluded in section 6 along with the proposed work for the future. 


\section{Background}

\subsection{Chat conversations for collaborative learning activities and personal ization}

Collaboration among students is an important element of learning. Collaborative learning experiences emphasize the concept of activities [2]. Performing any learning activity requires working with other learners. The interaction of learners via discussions and sharing contents take place through different online services [11].

Existing online services for discussion and collaboration like social media tools (facebook, twitter, ...etc) are varied in features and structure. Despite the availability of generating different types of annotations using existing services, it is more valuable to use a structured tool. The structured tool helps to provide more concise and easy to analyze annotations. Such a tool can enrich the chat conversation with features to generate and store the different types of annotations in a structured manner.

The chat conversations generated during collaborative learning task is a very valuable source of information for personalization purpose. Such conversations might contain information about the domain under discussion as well as information about learners' characteristics. To extract this information, there is a need to construct the domain ontology from the concepts or terms mentioned during discussions and relationships between them.

\subsection{Mining chat conversation}

With the aid of data mining and proper analysis of chat conversation, the valuable hidden information about learners and discussed topics can be attained [1], [2]. Chat mining as an application of text mining deals with the text or messages extracted from chat conversation [12] took place between actors. The discovery of knowledge or information (mining) from chat conversations can be performed by different techniques. Considering text mining, natural language processing (NLP) techniques must apply to understand natural language (text shared via SM) by the computer to create meaningful and structured information [13]. Modern NLP libraries use machine learning techniques like genetic algorithms, neural networks and support vector machines [14]. They can process a sentence, a paragraph, or an entire page of natural text and perform many operations such as tokenization, chunking, sentence segmentation, named entity extraction and parsing [6]. Text Mining includes tasks like; text categorization, text clustering, concept entity extraction (identify domain-specific terms), sentiment analysis, and entity relation modeling [15].

\subsection{Domain ontology}

Ontology has been used in the e-learning field in various ways depending on the task it serves [16]. For example, ontology can be adopted in the area of personalization by describing learner's information or profile including tracking learner's per- 
formance and selected learning resources. Ontologies can also describe a course content as a representation of course [17].

There are many existing definitions for ontology based on the application contexts and applied communities. For the purpose of this study, we are adopting the definition suggested in [18] which states that "An ontology defines the basic terms and relations comprising the vocabulary of a topic area as well as the rules for combining terms and relations to define extensions to the vocabulary". According to this definition, identifying the vocabularies representing the concepts related to the domain and the relations between the concepts is very important to construct the ontology. As social media involved and due to the need for semantic representation for data, an ontologybased model is incorporated for collecting and managing the data. Ontology is best suited for information extraction from text and chat conversations [19].

\subsection{Semantic relations}

Semantic relations are commonly used in most systems to represent knowledge and process information with reasoning features. For natural language processing (NLP) applications, acquisition, description, and formalization of semantic relations are required [20]. In order to identify the semantic relations, there is a need to find linguistic means exist in the texts. Normally the target relation to be discovered is a defined named relation expressed in the text either explicitly or implicitly.

There are different types of predefined named relations which can hold between concepts. When considering collaboration and pedagogical perspective, we should be able to define the relations which might exist among the components of learning activity tasks. The activity mainly consists of three main components which are: the main concept of the learning activity, the terms of concepts related to/part of the main concept and the resources or learning objects explaining those terms of concepts [2]. The types of semantic relations can describe a hierarchical relationship between concepts like Part-whole (PartOf), Kind-of, Has-a and Is-a relations [4]. They can also model a semantic connection like Hyponym and Hypernym relations or causality connection like Cause-Effect relation [20]. In addition to a pedagogical relation like Prerequisite which describes an order or association relationship [17].

These relations can be extracted using the rule-based technique. The extraction rules specify a pattern by capturing the relations that identify specific lexical elements in a text such as keywords [3]. The extraction and description of relations from textual data like chat messages require applying some text mining and algorithms [20].

\section{Related Work}

Mining chat conversation in general and in education, in particular, has been targeted by many researchers. For example, the authors in [21] developed a text mining tool which can identify patterns of interactions socially and semantically. The analysis of the chat conversations aiming at identifying the discussed topics and people in- 
volved in each topic. The tool also recognizing peers who are interacting with each other.

Another approach proposed by [19] presents an ontology-based chat logs analyses. The approach integrates knowledge processing mechanism with natural language processing and discourse analysis based on Bakhtin's concepts. The approach aims at detecting the chat topics, the threads of discussion and the important utterances. In addition, it visualizes the learners' contributions as a graph representation.

Aiming at predicting the students' final performance from online discussion participation, the researchers in [22], proposes the use of different data mining approaches like collecting data from the Moodle forum (twice during the course), pre-processing of data, predicting (classification and clustering) and interpreting by applying classification models, clustering models and mining association rules. This approach used only the messages which contain contents related to the course subject to improve the accuracy of classification.

The mining approaches have also been applied in [23] to analyze MOOC discussion forum messages. This analysis is aiming at identifying cognitive learning information. The researchers proposed a model to link the messages in MOOC discussion forums to a learning taxonomy. This model is targeting the identification of the different types of learning interactions taking place in forum conversations.

A text mining approach to constructing a domain ontology for representing the educational domain is proposed in [24]. The purpose of the domain model is to help students in making the choice of their desirable concepts. The suggested work focuses on modeling rich domain based on relationship types. The approach targeted the extraction of domain-oriented terms (object, attributes, entities, data models, the occurrence of terms) using different sources like text, web pages or databases. Examples of relationship discovery are is-a, Has part, and Has-subpart.

Recently a work carried out in [25], focused on analyzing chats conversations generated between teachers and learners as an example of educational and argumentative dialogues. It aims at identifying the most important discussed threads from the extracted scientific threads.

Despite the efforts carried out by researchers in the field of mining chat conversation throughout the years, we totally agree with the issues pointed out in [10], which states that there is an ignorance of taking full advantages from the Social Web during the collaboration support for learning. More specifically the lack of utilization of the actual content of the generated messages during collaboration. Thus, the research presented in this paper focuses on the best utilization of the generated content to construct the domain model and understand the learner's characteristics. Consequently, provide personalization feature for the individual learner. To achieve this task, the proposed mining model in this paper aims at structuring a predefined domain ontology from chat conversations or discussions during collaborative learning activity by identifying the concepts and semantic relations exist among them. To provide the personalized package, collaborative filtering task will be performed to identify two parameters which are learning style and knowledge level. The description of the proposed model is presented in the following section (section 4) 


\section{$4 \quad$ Mining Model for Semantic Relation Extraction and Ontology Representation}

This section discusses the mining model followed to extract information from the chat conversations (see Figure 1). The model consists of four main activities which start with a collection of chat messages either from one collaboration tool or multiple tools. Next, performing pre-processing as a mean of text mining technique to clean the collected data. Then, building the domain ontology based on the extracted concepts and semantic relations from the preprocessed chat messages. Finally, identify two learner's characteristics (knowledge level \& learning style) as a mean of the collaborative learning task. All these activities/tasks will be discussed below.

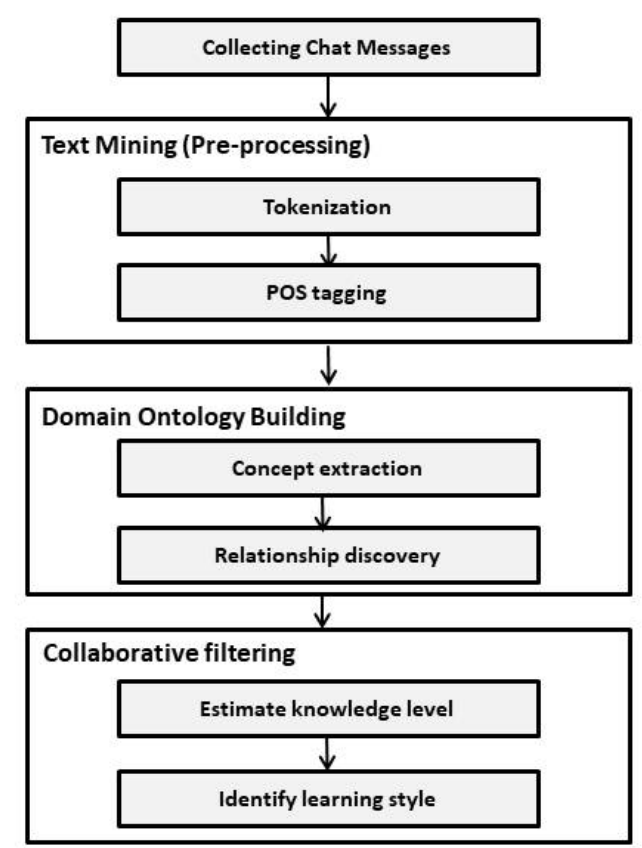

Fig. 1. Mining Model for Extracting Information from a Chat Conversation

\subsection{Collecting chat messages}

The proposed approach is applicable to data collected from one or many collaboration tools. For collecting data from multiple tools (SM), an aggregation and mapping mechanism should take place to generate a single file aggregation data from different tools [26]. The suggested aggregation and mapping methodology are based on the concept of matching operation. To perform any matching operation, the files (JSON/XML) extracted from social media platforms (using API) will be compared to find the similarity in the attributes using the matching mechanism. To do so, an input 
alignment needs to be identified. In the matching process, external resources such as WordNet is used to find the gloss of the synonym attributes. In addition to the external resources, defined matching parameters like (weight and threshold) is required to identify the degree of similarities between attributes. The full details about this aggregation are presented in the previous publication [2]. However, for this paper, the data is collected from one application (PerLCol collaborative learning tool) as it is going to be discussed in the experiment section. The data collected from the tool are the form of different annotations like comments, question/answer, rating (expressed opinion) and shared resources.

The collected data needs to be pre-processed to be ready to extract useful information. The preprocessing of data includes two main tasks to complete which are tokenization and POS tagging.

\subsection{Pre-processing}

Tokenization: The first step in the pre-processing task is the tokenization of the text. Tokenization is the task of splitting the input text into very simple units, called token. This is a required step in any linguistic processing application. Tokenization involves sentence boundary detection, simple white space identification, and name recognition. In addition, tokenization for social context also requires treating hashtags, URLs, and emoticons [27][13]. The TwitIE tokenizer follows Ritter's tokenization scheme [28]. This scheme treats abbreviations (e.g. RT, ROFL) and URLs as one token each. Hashtags and user mentions are two tokens if they occur in a sentence, plus a separate annotation HashTag covering both [GATE].

When considering the social media tools a source for the collected data, the existence of linguistic noise is high. Thus, identifying the correct words/forms of the orthographic errors is important before moving to the next task which is the POS tagging. GATE is using Han et al approach as a lookup dictionary to normalize the text. The dictionary includes well known correctly-spelled terms, and refer to invocabulary (IV) and out-of-vocabulary (OOV) terms with respect to this dictionary. For example, the misspelled words like "2morrow", info, abt, u, etc, are corrected as Tomorrow, Information, About, You respectively.

For example, the message "i need more info abt waterfall model" will be tokenized and normalized as shown below.

\begin{tabular}{|c|c|c|c|c|c|}
\hline I & Need & More & Info & Abt & waterfall \\
\hline I & Need & More & Information & About & waterfall \\
\hline
\end{tabular}

POS tagging: The tokenized text is now ready to perform the POS tagging task. lexical-morphological analysis has to be done using a POS (Part-of-Speech) tool. POS specifies if a term is a verb, a pronoun, an adjective, an adverb, a noun. etc. TwitIE contains an adapted Stanford tagger trained on tweets tagged with the Penn TreeBank (PTB) tagset [29]. Extra tag labels have been added for retweets, URLs, hashtags and user mentions incorporating two existing POS taggers [28] and [30]. To improve the tagging, TwitIE is equipped with a gazetteer lookup and a normalizer [29]. Combin- 
ing normalization, gazetteer name lookup with the Twitter-specific POS tags, improve the tagging of the user-generated content on SM applications like Twitter.

$\begin{array}{ccccccc}\text { I } & \text { Need } & \text { More } & \text { Info } & \text { Abt } & \text { waterfall } & \text { Model } \\ \text { I } & \text { Need } & \text { More } & \text { Information } & \text { About } & \text { waterfall } & \text { Model } \\ \text { PRP } & \text { VB } & \text { JJ } & \text { NN } & \text { IN } & \text { NN } & \text { NN }\end{array}$

The above is an example of POS tagging for a comment written by a learner.

In case of question/answer annotation, the learner can ask a question like what about REQ phase? And other learners can answer the question. The idea behind using question/answer annotation is to understand the level of knowledge. As well as helping the learner to construct knowledge and clarify their doubts. The question now is how to use this type of annotation to estimate the knowledge level?

The idea is based on the concept of asking question resulted from a lack of understanding of a specific concept/s. Besides, answering question/s indicates a good level of understanding of the concept/s. Therefore, the more the learner is asking the question, the more helshe having doubts and concerns. In addition, the more the learner is answering the questions, the more he/she has knowledge.

Analyzing the $\mathrm{Q} / \mathrm{A}$ annotation requires extracting the used keywords (noun/verb) in the question to identify the related concept.

$\begin{array}{ccccc}\text { what } & \text { About } & \text { REQ } & \text { phase } & ? \\ \text { what } & \text { About } & \text { Requirement } & \text { phase } & ? \\ \text { WP } & \text { IN } & \text { NN } & \text { NN } & \text { SYM }\end{array}$

The given answers need to be analyzed to identify the related concept and level of knowledge shared by the learner. The analysis of the answers is similar to the one for comments. Analyzing the questions is aiming at identifying the type of question and the related concept by recognizing the (noun/verb). As for the answers, the analysis is similar to the comment annotation. The target in this analysis is to identify the concept/s contained in the statement. The output of this step is cleaned well-structured annotations ready to be analyzed to build the domain ontology. To transform and build the domain ontology, two tasks need to be performed, the concept extraction and relation discovery. These tasks are important to construct the structure of the domain ontology which consists of concepts and relations between them.

\subsection{Domain ontology building}

After preprocessing the annotations, tasks related to extracting the concepts and discovering the relationship between them is needed to build the domain ontology. This task is aiming at identifying relevant triples (pairs of concepts connected by a relation) which can be integrated into the predefined ontology. The following sections discuss in details the techniques for concept extractions and relations discovery.

Taking the example of performing a collaborative learning activity, the semantic relations for this type of task is shown in Figure 2. This learning activity has a list of vocabularies representing terms or concepts related to the LA. This relation called 
HasPart, which means that the term/concept is part of the LA. These concepts/terms can be also related to each other using the IsRequiredBy relation. Another connection is the HasResource relation where each concept/term connect to a learning object (LO) using the HasResource relation. Identifying these concepts and relations will be using concept extraction and relationship discovery as explained below.

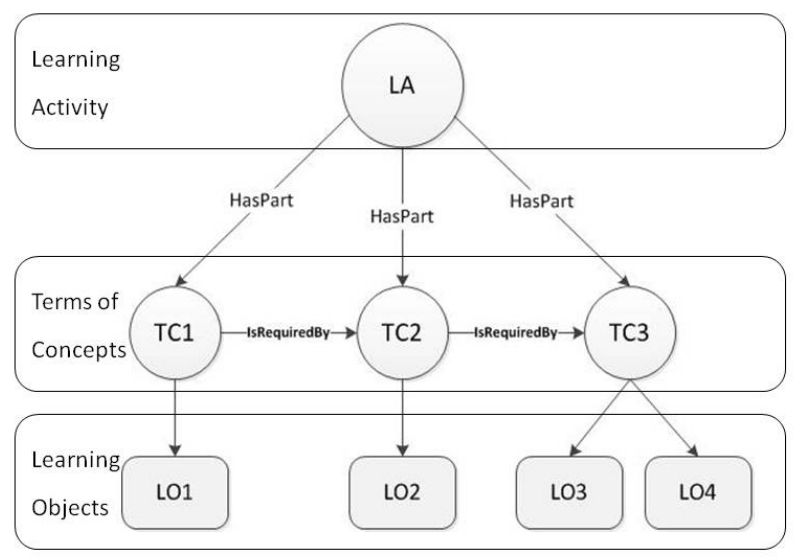

Fig. 2. Semantic Relations in Learning Activity Model

Term/concept extraction: The goal of this step is to select domain terms, which are relevant to the domain description. There are two main concepts need to be recognized in this step (Named Entity \& Terms). Entity Recognition is all about recognizing and classifying key Named Entities and terms in the text. Named Entity is a Person, Location, Organization, Date, etc. Whereas, the term is a key concept or phrase that is representative of the text. Term extraction is a very crucial step in building the domain model. The determination of the terms is depending on the list of terms defined in gazetteer lookup and the tagged nouns in POS tagging. POS Tagging is one of the important features which can be adopted for concept extraction as a language dependent feature [31], [32]

As discussed before in POS tagging, the concept extraction requires identifying the terms which are represented as NN (nouns). Besides, to recognize these terms, a lookup mechanism is performed using gazetteer. Gazetteer is one of the components in the GATE. This component consists of predefined lists of terms such as cities, organizations, days of the week, etc. It not only consists of entities but also of names of useful indicators, such as typical company designators (e.g. 'Ltd.'), titles, etc. The gazetteer lists are compiled into finite state machines, which can match text tokens.

To perform the task of concept extraction, the terms which are related to the domain (main concept/terms of concepts) should be added to the gazetteer. For example, in the case of the waterfall model, the terms to be added are "waterfall model, requirements, analysis, design, implementation, testing, maintenance". 
For example, the messages shared by a learner as "The model has six processing activities, requirements, analysis, design, implementation, testing \& maintenance"

Looking up for the existence of these terms (nouns) in the generated message based on the list in the gazetteer. See sample messages below.

\begin{tabular}{|l|}
\hline I think this model works well for smaller projects where requirements are very well understood \\
\hline I need more information about waterfall model \\
\hline $\begin{array}{l}\text { The model has six processing activities, requirements, analysis, design, implementation, testing \& } \\
\text { maintenance. }\end{array}$ \\
\hline We should start with requirement, because design is to allocate requirement \\
\hline Let's start thinking about design architecture \\
\hline Hi all, can anyone help me to know the concepts in design phase of waterfall model \\
\hline What about requirement phase? \\
\hline Can we start with the design phase? \\
\hline
\end{tabular}

Semantic relation discovery: Relation extraction from text aims at detecting and classifying semantic relations between entities according to a predefined entity and relation type system or an ontology [33]. In our case, an ontology has been defined.

Discovering relationships between extracted concepts/terms is refers to identifying linguistic or conceptual relationships among their terms/concepts [20]. Such extraction depends mainly on verbs appears on the text/messages. Therefore, the rulebased/pattern-based technique is used to identify the relations.

Relationship discovery step is very important in linking the identified terms together and building the domain. There are many techniques which could be used to discover the connections between the concepts discussed during collaborative e-learning activities using SM applications. These connections can be discovered from the text using some statistic and linguistic analysis. This is referring to identifying or extracting the linguistic relationships between terms/concepts. Such extraction depends mainly on verbs appears on the text/messages. Therefore, the rule-based/pattern-based technique is used to identify the relations.

As mentioned before there are three relations need to be discovered which are HasPart, IsRequiredBy, and HasResource for the predefined ontology building. In our approach, we are using a rule-based concept relation extraction/discovery which will be discussed in the following section.

- Rule-based concept relation discovery

Rule-based information extraction uses specific rules that describe patterns to be matched. Discovering the relationship between concepts is performed using the rulebased technique to extract a triple pattern. The triple is linking between two concepts/terms in the form of nouns and a relation in the form of a verb $<$ noun, verb, noun $>$. For example, referring to our domain ontology, the target will be two triples which are; <main concept (LA), relation, term of concept $>$ and $<$ term of concept, 
relation, term of concept $>$. If such triples found, then it is an indication of an existing pattern. The nouns in the triple represent the identified terms and the verb represent the type of relationship between them. The rules are formulated as follow:

Definition 1. The associations or relations between the main concept (LA) and term of concept (TC) is identified by HasPart relation. The concepts LA and TC are said to be associated with each other if the type of relation HasPart is expressed as ART.User/Owner relation in ACE2004 evaluation. This relation also represented as "own", "have", "hold" names in verbnet1 (own-100.1) with "has-possession" as one of the values.

For example, in the message "The model has six processing activities, requirements, analysis, design, implementation, testing \& maintenance" (See figure 3)

- Has expresses an ART.User/Owner relation in the ACE evaluation

- The linked entities/terms are e.g (model \& activities), (model \& requirements) .... etc.

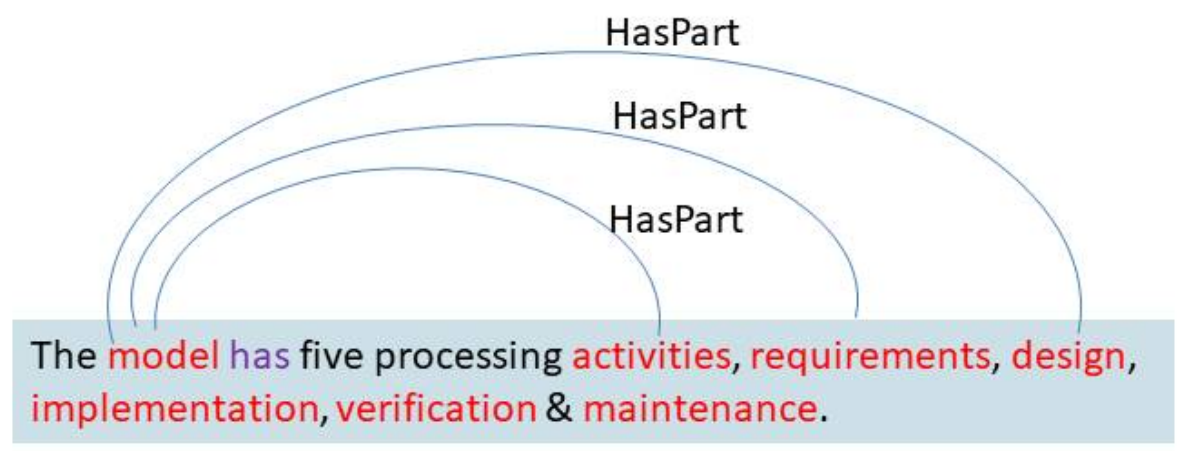

Fig. 3. An Example of Relation (HasPart) to be Discovered in a Chat Text.

Definition 2. The associations or relations between TCs (TCi and TCi+1) is identified by IsRequiredBy. The concepts TCs are said to be associated with each other if the type of relation (IsRequiredBy) is expressed as GPE-AFF. Based-In relation in the ACE2004 evaluation.

For example, in the message "We should start with requirements, coz design is to allocate requirements" (See figure 4)

- Allocate express a GPE-AFF. Based-In relation

- The linked entities/terms are design \& requirements

\footnotetext{
${ }^{1}$ https://verbs.colorado.edu/verbnet/
} 


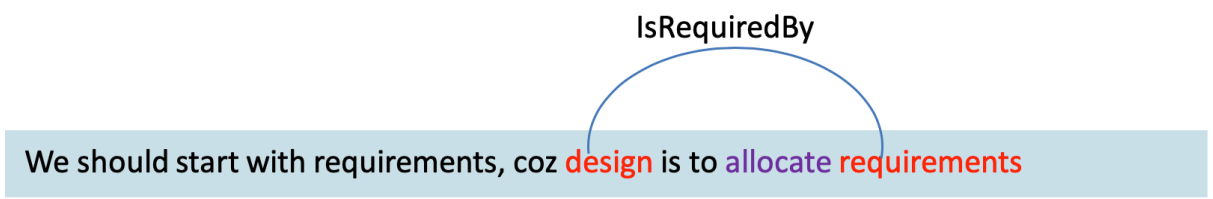

Fig. 4. An example of Relation (IsRequiredBy) to be Discovered in a Chat Text.

Definition 3. The associations or relations between Term of Concept (TC) and Learning Object (LO) is defined by HasResources relation. The LO explains the TC/s if the metadata (Context....) of the LO covering the TC.

To differentiate between the relations, Table 1 describes the relations.

Table 1. Lexical Relations Description

\begin{tabular}{|c|c|c|}
\hline Relation Type & Description & Example \\
\hline HasPart & $\begin{array}{l}\text { Part of relation } \\
\qquad \begin{array}{l}P a r t O f(L A, T C) \rightarrow T C_{i} \\
\quad=\left\{t c_{1}, t c_{2} \ldots t . t c_{n}\right\} \text { and } T C_{i} \subseteq L A\end{array}\end{array}$ & $\begin{array}{l}<\text { main concept (LA), relation, term of } \\
\text { concept }> \\
\{\text { Token.string =="has" }\}\end{array}$ \\
\hline IsRequiredBy & $\begin{array}{l}\text { An order relation, a prerequisite relation } \\
\quad \text { IsRequiredBy }(T C, L) \rightarrow C \\
\quad=\left\{t c_{1}, t c_{2} \ldots . t c_{n}\right\} \text { and } L=l(i, j) \\
\quad \rightarrow t c j \text { is prerequisite for } c i \text {, where }(t c i, t c j) \\
\quad \in T C\end{array}$ & $\begin{array}{l}\text { <term of concept, relation, term of } \\
\text { concept }> \\
\{\text { Token.string =="allocate" }\}\end{array}$ \\
\hline HasResources & $\begin{array}{l}\text { Explain relation } \\
\quad \text { HasResources }(L O, T C) \rightarrow L O \text { Explains } T C\end{array}$ & $\begin{array}{l}<\text { term of concept, LO }> \\
\{\text { Metadata value=="matched" }\end{array}$ \\
\hline
\end{tabular}

\subsection{Collaborative filtering}

After the structuring of the domain ontology based on the concepts and relations extractions, the collaborative filtering task will take place. This task is targeting the identification of two learners' characteristics which are the learning style and knowledge level. These characteristics will be used as parameters to provide a personalized learning package.

- Learning Style Identification

The learning style identification is carried out by using an ontology-based Dynamic Bayesian Network (DBN) model to represent the relationship between the learning style and preferable learning object. The model also obtains the learner's opinion more than one time by using a time slice to make the indication of learning styles more accurate. Learners' opinion and expressed emotions have an effect on their performance and the learning process in general [34]. As discussed in [1], the FelderSilverman's Model [35], is used for modeling the learning style by classifying them into four dimensions: processing (Active/Reflective), perception (Sensory/Intuitive), input (Image/Verbal), and understanding (Sequential/Global). To determine the learning style of any learner, there is a need to extract information related to the learner's preferable format of learning objects as they have a direct relationship with the learning style identification. Based on the preferable learning objects, the learning style can 
be identified. For example, if the learner likes to view audio and video media, then this is an indication that he/she belongs to verbal learning style.

The grouping of learners is by considering the social dimension and interaction concepts [36] [37]. Based on this dimension, the learners will be categorized into three groups (participatory, collaborative, and independent). These three groups are reflecting the learning styles which are identified based on the characteristics of each group. The grouping dimensions of the learners according to the identified learning style are defined as follow:

- Participatory: \{Visual, Sensory\}

- Collaborative: \{Active, Global\}

- Independent: \{Verbal, Intuitive, Reflective, Sequential\}

The following table (Table 2) elucidates the preferable learning objects in each group.

Table 2. The Preferable Learning Object/s Based on The Learning Style Belong to the Learner Social Grouping.

\begin{tabular}{|l|l|}
\hline \multicolumn{1}{|c|}{ Learner Social Grouping } & \multicolumn{1}{c|}{ Preferable Learning Object Type } \\
\hline Participatory & Image, Video, Animation, Hypertext, Chat Application \\
\hline Collaborative & Chat Application \\
\hline Independent & Audio, Video, Text, Hypertext \\
\hline
\end{tabular}

- Knowledge level estimation

The Knowledge Level modeling imitates the structure of the domain model. The overlay modeling approach is used for modeling student knowledge based on a domain model which is constructed as Domain knowledge ontology. For each domain concept, an overlay model stores estimated the level of knowledge. Overlay Modeling: describe user characteristics, e.g. "knowledge of a user" with respect to "ideal" characteristics.

To estimate the knowledge level of each learner based on the predefined domain ontology, the chat messages participated by the learners will be utilized. For example, the learner's contribution like the number of comments shared and/or the number of questions/answered participated are used for the measurement of knowledge level parameter.

In this study, the authors adopt the concept of measurement suggested by [38] to define the content richness score. The idea is to find how rich or strong the contribution in relation to the concept under discussion.

$$
K L_{S}=\frac{\sum_{i=1}^{n} m c i}{n * \max (m c)}
$$

- $\mathrm{s}$ represents the student, mci represents the value/weight of the content in the message $\mathrm{i}$ (relatedness to the concept) as describes in Table 3. 
Table 3. The Weighting of the Message Content

\begin{tabular}{|l|l|l|}
\hline Weight & \multicolumn{1}{|c|}{ Meaning } & \multicolumn{1}{c|}{ Description } \\
\hline 0 & Not related & No words related to the concept found in the message. \\
\hline 1 & Related & 1 to 2 words related to the concept found in the message \\
\hline 2 & Very related & More than 2 words related to the concept found in the message. \\
\hline
\end{tabular}

- $\mathrm{n}$ is the total number of messages on a particular topic shared by $\mathrm{s}$,

- $\max (\mathrm{mc})$ it is representing the maximum possible value of message content quality.

- KL score can range from 0 to 1 .

- 0 indicating a low level of knowledge since no contribution by that learner/student on the specific topic.

-1 means a high level of knowledge and excellent engagement by that learner.

There are three levels of knowledge to be identified based on the measurement results as shown in the following table (Table 4).

Table 4. The Levels of Knowledge Based on the Measurement Results

\begin{tabular}{|l|l|}
\hline \multicolumn{1}{|c|}{ Knowledge level measurement result } & \multicolumn{1}{c|}{ Knowledge level } \\
\hline $0.00-0.33$ & Poor \\
\hline $0.34-0.66$ & Medium \\
\hline $0.67-1.00$ & High \\
\hline
\end{tabular}

The estimated knowledge level and identified learning style will be used to provide a personalized learning package. The package consists of the learning object/s for the term of concept required to learn based on the learning style.

\subsection{Personalization Package}

The personalization package is performed based on the Knowledge level and the grouping dimensions. The grouping is categorized according to identified learning style and their preferable learning object format.

According to the identified domain model (see Figure 5b), the learner should be aware of the term of concepts belong to the learning activity (waterfall model). Measuring the knowledge level of each learner will help to identify the next term of concept to be aware of. For example, if the identified knowledge level of a learner in a particular term of concept is poor and it is a prerequisite for the next concept, then the learner should be provided with learning object/s related to the current term of concept. However, if the identified level is medium or high, then the learning object/s should belong to the next term of concept in the domain model. Identifying the appropriate learning object/s is according to the learner's grouping of the learning style. For example, learners who belong to the participatory group are either Visual or Sensory learning styles. Thus, this group prefers learning objects in the formats of image, video, animation, hypertext and chat application. Therefore, the personalized learning package will be consisting of a learning object in one or more of these formats. The algorithm to perform the personalization is shown in Figure 5. 


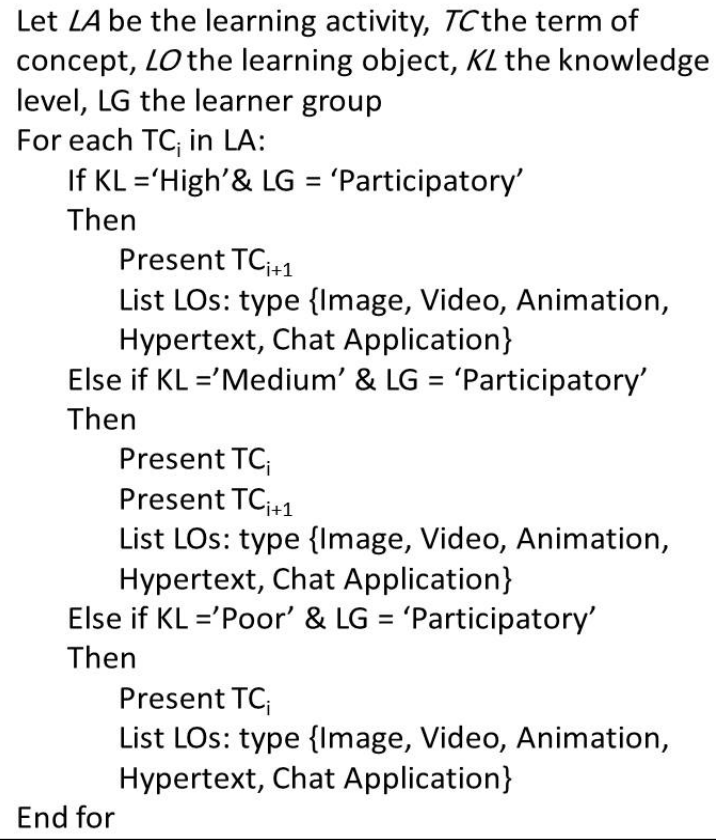

Fig. 5. The Algorithm to Perform the Personalization Task.

\section{Experiments and Discussion}

For evaluating the proposed mining model, an experiment conducted for 24 learners registered in the department of information technology at Ibri College of Technology, Oman. The students are studying introduction to software engineering course at diploma level. The controlled experiment was designed previously by the course teacher based on a collaboration scenario for learning activity. The learning activity is related to one of the system development lifecycle (SDL) models which is "the waterfall model". The main concept (waterfall model) has five terms of concept (Requirements, Design, Implementation, Verification, maintenance). The detailed collaboration scenario and the carried analysis using the model are given below.

\subsection{Collaboration scenario}

To perform the collaborative learning task, the PerLCol collaborative learning tool has been used. The collaboration tool enriches with similar features of the social media platforms. As illustrated in Figure 6, the tool has four main annotation types to facilitate collaboration between learners. They are described below:

- comment, textual remarks or note related to a content or the topic under discussion. The textual remarks can also be an expressed opinion related to the content. 
- $\quad$ rating (like/dislike), an opinion expressed related to content. This rating involves two opinions either like or dislike the shared/selected content.

- question/answer, a question raised by a learner or an answer given by another learner/s.

- share external resources, external resources related to a concept.

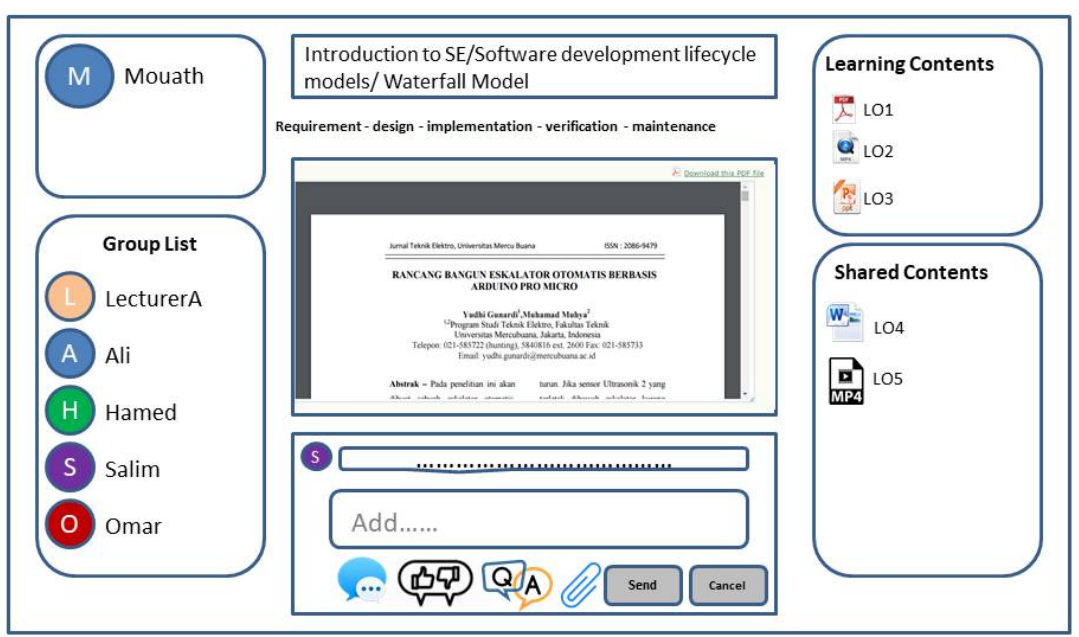

Fig. 6. PerLCol Collaborative Learning Tool

The tool allowed learners to be grouped based on the allocated learning activity. The discussion will be opened for learners until the deadline decided by the educator as a duration for performing the learning activity. During discussion and collaboration, learners will be provided with learning contents uploaded by the educator. Besides, they can also share external learning content/resources. The main features available for learners apart from the sharing of resources are: commenting on discussed concept/topic, expressing their opinion on learning resources (textual remarks or rating icon). In addition, the feature of asking or answering a question is provided separately to form or structure the process of asking or answering a particular question. The present of educator throughout the process of collaboration is supported by the tool. This way the educator can play the role as a facilitator to guide learners and monitor the collaboration process.

The available annotations are considered as sources of information to understand the characteristics of learners as well as building the domain model under discussion. To do so, these annotations should be collected and gone through some text mining techniques. Among the available annotations, comment and question/answer need to be preprocessed for further analysis and extract useful information related to the domain model under discussion and preferences of learners involved in the discussion. Besides, information related to the level of knowledge of each learner can also be extracted from these annotations. Sample chat log displaying shared comments during the discussion related to waterfall topic (learning activity) is shown in Figure 7. 


\begin{tabular}{|c|c|c|c|c|c|}
\hline comment id & user id & topic id & comment & Term of concept id & DateTme \\
\hline 83 & 24 & 32 & I think this model works well for smaller projects where requirements are very well understood & 51 & 3/24/2019 15:37 \\
\hline 84 & 24 & 32 & i need more information about waterfall model & 52 & $3 / 24 / 2019$ 15:37 \\
\hline 85 & 30 & 32 & The model has five processing activities, requirements, design, implementation, verification \& maintenance. & & $3 / 24 / 201915: 46$ \\
\hline 86 & 30 & 32 & We should start with requirement, because design is to allocate requirement & 48 & $3 / 24 / 201915: 48$ \\
\hline 95 & 28 & 32 & Let's start thinking about design architecture & 49 & $3 / 24 / 201915: 48$ \\
\hline 97 & 18 & 32 & hi all, can anyone help me to know the concepts in design phase of waterfall model & 49 & 3/24/2019 22:02 \\
\hline 98 & 28 & 32 & Hey I can't get waterfall model & 48 & $3 / 24 / 201922: 14$ \\
\hline 99 & 32 & 32 & Meas a student I don't like the normal way of teaching (daily rotten) & 48 & $3 / 24 / 201922: 14$ \\
\hline 100 & 28 & 32 & great! you are distinguished in offering and presenting ideas. & 50 & 3/24/2019 22:14 \\
\hline 102 & 28 & 32 & i need more deceleration in design & 52 & 3/24/2019 22:17 \\
\hline 106 & 118 & 32 & how people actually work with the data & 48 & $3 / 25 / 201910: 27$ \\
\hline 107 & 101 & 32 & Requirements is important information about the project that will help you to do the project well. & 48 & $3 / 25 / 201910: 28$ \\
\hline 108 & 114 & 32 & The requirement is a description of the & 48 & $3 / 25 / 2019$ 10:28 \\
\hline 109 & 109 & 32 & Desigg is very simple to understand and easy.... & 49 & $3 / 25 / 201910: 28$ \\
\hline 114 & 99 & 32 & This diagram above shows the step one by one for when we nee to collect the requirements from the customer. First observe & 48 & $3 / 25 / 201910: 32$ \\
\hline 115 & 100 & 32 & the requirements tell me about the Scoop they have the most successful database & 48 & $3 / 25 / 201910: 35$ \\
\hline 116 & 110 & 32 & code design use programming language & 50 & $3 / 25 / 201910: 35$ \\
\hline 117 & 113 & 32 & ways to collect information & 48 & $3 / 25 / 201910: 35$ \\
\hline 118 & 105 & 32 & The requirement is a description of the needs & 48 & $3 / 25 / 2019$ 10:35 \\
\hline 120 & 104 & 32 & the requirements is description of a lot of condition that define for the system. & 48 & $3 / 25 / 201910: 35$ \\
\hline 121 & 102 & 32 & I don't like because more information and difficult word & 48 & $3 / 25 / 201910: 35$ \\
\hline 126 & 99 & 32 & I like it. it is easy to understand fast. .it shows the general model of design process (design input & 49 & $3 / 25 / 2019$ 10:35 \\
\hline 128 & 102 & 32 & the user create the plan to organize and order when do project & 49 & $3 / 25 / 2019$ 10:39 \\
\hline 129 & 104 & 32 & the third step of waterfall is implementation & 50 & $3 / 25 / 201910: 39$ \\
\hline 130 & 109 & 32 & This style for implementation is very clear to understand. . & 50 & $3 / 25 / 201910: 40$ \\
\hline 131 & 104 & 32 & verfication can you check if any error & 51 & $3 / 25 / 201910: 41$ \\
\hline 132 & 108 & 32 & the requirement is awav to collect the information for anv proiect. the interview is one awav to collect requirement mean is $m$ & 48 & $3 / 25 / 201910: 42$ \\
\hline 133 & 117 & 32 & it is easy and good because get more information about the topic & 50 & $3 / 25 / 201910: 42$ \\
\hline 134 & 110 & 32 & in Maintenance is can do the change to reused & 52 & $3 / 25 / 201910: 42$ \\
\hline 135 & 100 & 32 & design sample becuase it is do the requirement befor then design. & 49 & $3 / 25 / 201910: 43$ \\
\hline
\end{tabular}

Fig. 7. A Chat Log for the Shared Comments Annotation.

This collaboration tool with the similar features of social media applications facilitates the discussions and provides a better and controlled way to manage the collaboration process. The generated chat conversations using this tool is the main source to build the domain model as well as understanding the characteristics of the participated learners. The targeted characteristics are the learning style and knowledge level. Said characteristics are the parameters to provide personalized learning package for the learners. To extract the required information from the chat conversations, the proposed mining model is used as discussed below.

\subsection{Data collection and analysis}

Data collection and pre-processing: The chat conversation has been collected after the discussion period completed. The total number of annotations generated by users was 278 . They have been categorized into three groups according to the annotation type. These groups are:

- Explanation comments, this includes discussion about the concept.

- Opinionated comments, this includes messages expressing opinions either positive or negative like nice, good, bad...etc.

- Question \& Answer annotations, this includes messages contains questions and answers for these questions. 
The obtained data for the study (messages) was collected and went through the activities based on the mining model proposed in this paper. The sample collected data categorized into three groups according to the annotation type. These groups are:

- Explanation comments, this includes messages like:

\begin{tabular}{|l|}
\hline I think this model works well for smaller projects where requirements are very well understood \\
\hline I need more info abt waterfall model \\
\hline $\begin{array}{l}\text { The model has six processing activities, requirements, analysis, design, implementation, testing \& } \\
\text { maintenance. }\end{array}$ \\
\hline We should start with req, coz design is to allocate req \\
\hline Let's start thinking about design architecture \\
\hline
\end{tabular}

- Opinionated comments, this includes messages like:

\begin{tabular}{|l|}
\hline \multicolumn{1}{|c|}{ the file is excellent } \\
\hline I prefer image \\
\hline great! thx \\
\hline Very nice \\
\hline Hey I can't get waterfall model \\
\hline Thank u Dr. It will help me to understand the model \\
\hline
\end{tabular}

- Question \& Answer annotations, this includes messages like:

\begin{tabular}{|l|}
\hline \multicolumn{1}{|c|}{ hi all, can anyone help me to know the concepts in design phase of waterfall model } \\
\hline what abt REQ phase? \\
\hline any suggestions on good materials? \\
\hline can we start with the design phase? \\
\hline yea, you can go for SE book by Pressman \\
\hline u can also read this file, it has detailed info \\
\hline
\end{tabular}

The collected data has been pre-processed and cleaned based on the method discussed previously in section 4.

Domain ontology building: After the pre-processed of the collected data, we have been able to construct the domain ontology for the waterfall model from the shared messages using the proposed Rule-based concept relation discovery as discussed in section 4.3. The constructed domain model presented in Figure 8. The model is representing waterfall model concept and its phases as the terms of concepts in the ontology. 


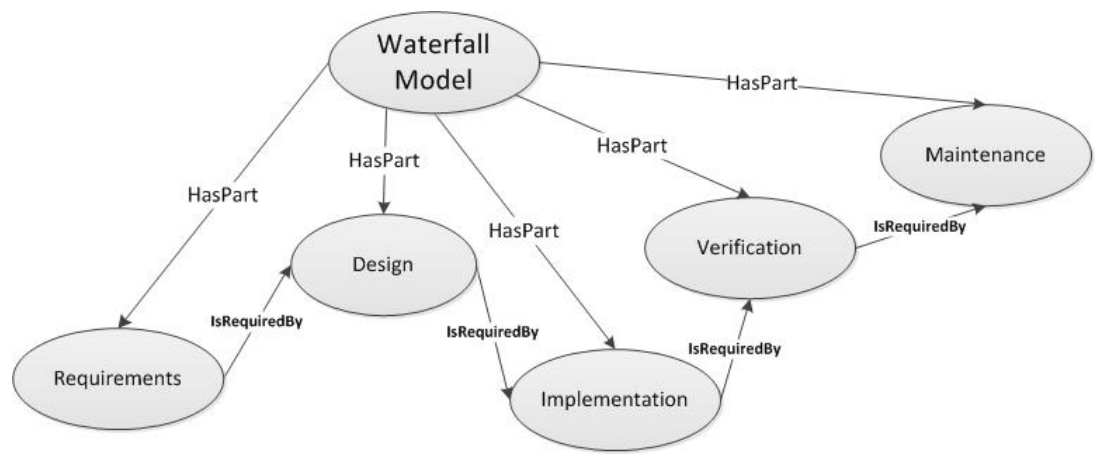

Fig. 8. Semantic Relations in Waterfall Model Learning Activity

Collaborative filtering: The next step is to perform the collaborative filtering task for indicating the learning style and knowledge level for the participated learners.

The identification of learning style is linked with the learning object preferences indicated by learners. To extract information related to the learner's preferences from chat conversation, opinion mining is the best technique to adopt. This technique helps to identify the preference of the learners in terms of the type of learning content rated via like icon or opinion expressed by a particular learner. Sentiment analysis as one technique in this field is used to identify the opinion of a learner in a particular learning object format. All the details can be found in our previous publication [1]. For example, the learner with the user_id \#116 from the group of learners participated in this study, gave his opinion on an image learning object as positive (100\%) which indicated by pressing the Like icon. Therefore, as the first indication, the learner's learning style is identified as either visual $(75 \%)$ or sensing $(25 \%)$ based on the relationship between learning object format and learning style. For the second entry, the same learner (\#116) has a positive opinion (100\%) on a hypertext learning object as expressed by (Very nice), which means $(50 \%)$ sharing with the previous opinion on the image. Consequently, the identification of the learning style updated as visual $(100 \%)$. The DBN models to represent the relationship between the learning style and preferable learning object obtained using a time slice (t1 \& t2) are depicted in Figure 9. 


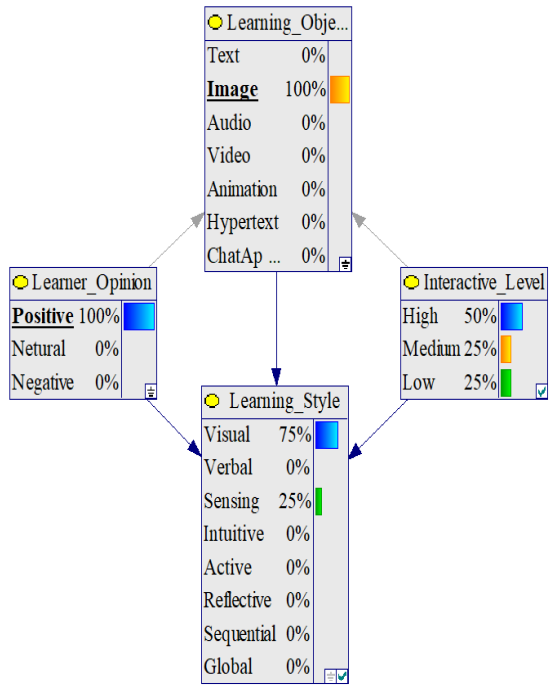

Fig. 9. 1a. DBN Model After Learner's Opinion (t1)

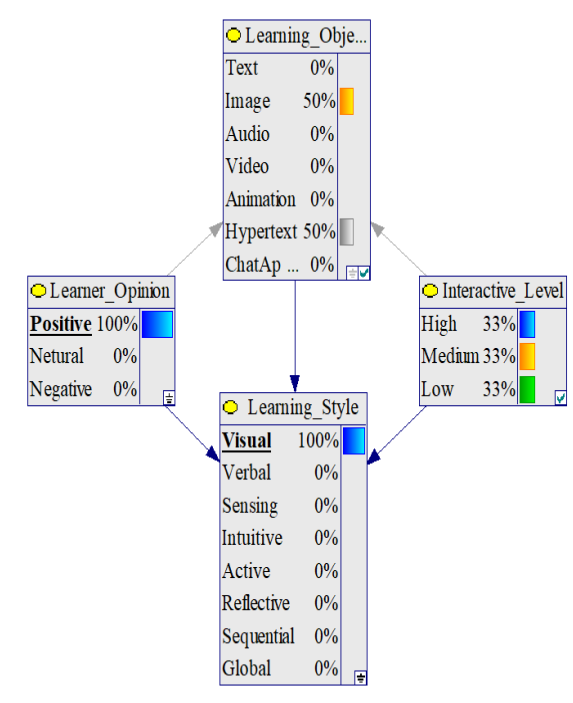

Fig. 9b. DBN Model After Learner's Opinion (t2)

The use of the time slice ( $\mathrm{t} 1 \& \mathrm{t} 2)$ indicates the accurateness of the learning style identification sine visual learner prefers both image and hypertext format as oppose to sensing who prefers image and animation. As a result, this learner (\#116) should be provided with a personalized learning package consist of $\mathrm{LO} / \mathrm{s}$ of the types image, hypertext, video, and animation.

The social grouping based on the preferable learning object is depicted in Figure 10 The result as shown in Figure 6.6 indicates that the majority of the students participated in the experiment filled under participatory social group (79\%). The remaining students are in an independent social group (21\%) while no students fit under collaborative social group. Which indicate that this group of students is not socially active, they can participate if they are giving the opportunity to contribute with their ideas but they can also work individually so comfortably [36]. 


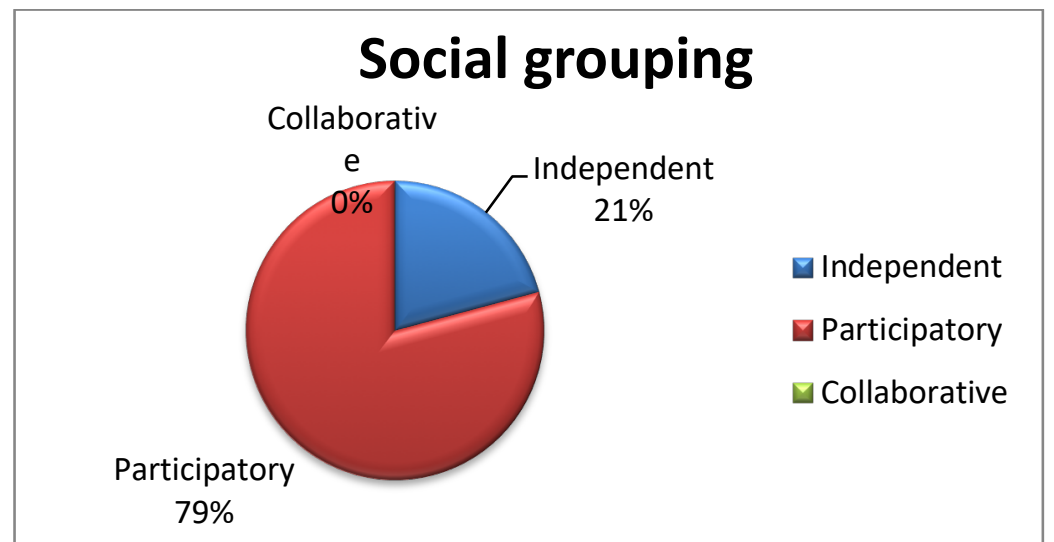

Fig. 10.Social grouping categorization

The selection of the learning objects is depending on the term of concept to be learned. The identification of the terms of concept is according to the measurement of knowledge level obtained and the relations between terms in the domain model. For example, for the activity related to the waterfall concept as shown in Figure 8, the estimated knowledge levels related to the main concept for the participants are graphically represented in Figure 11. This measurement is calculated using the method discussed in section 4 previously (Knowledge level estimation).

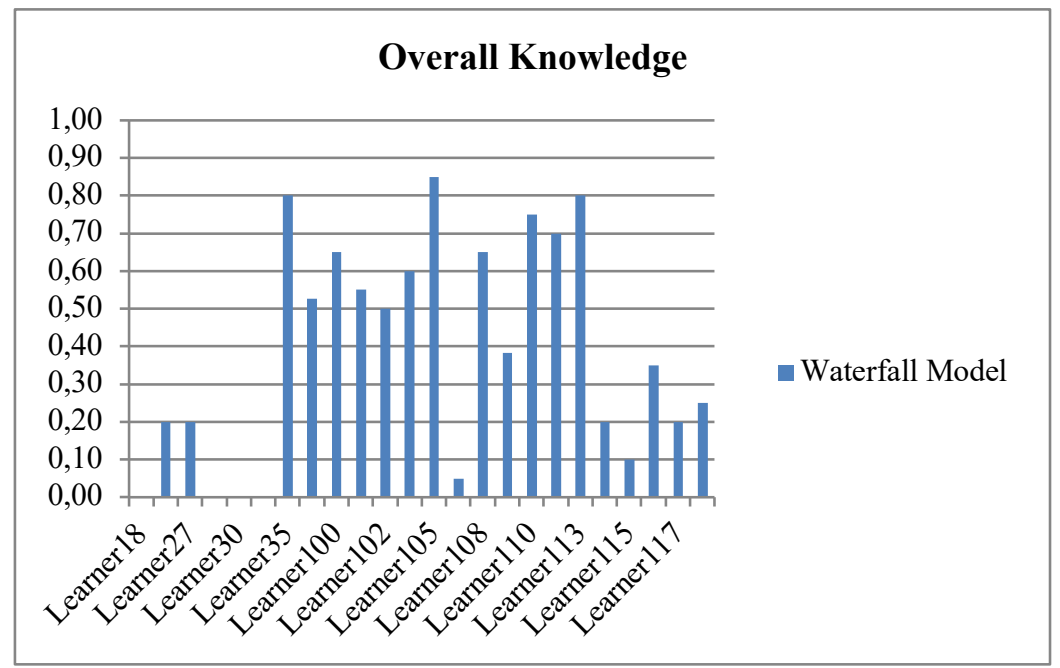

Fig. 11.Graphical Representation of Knowledge Level Estimation

The estimated knowledge for every term of concept and overall knowledge of the group on the waterfall model is represented in Figure 12. This representation indicates 
the level of knowledge categorization (Low, Medium, High) based on the definition of knowledge level measurement discussed previously in section 4 .

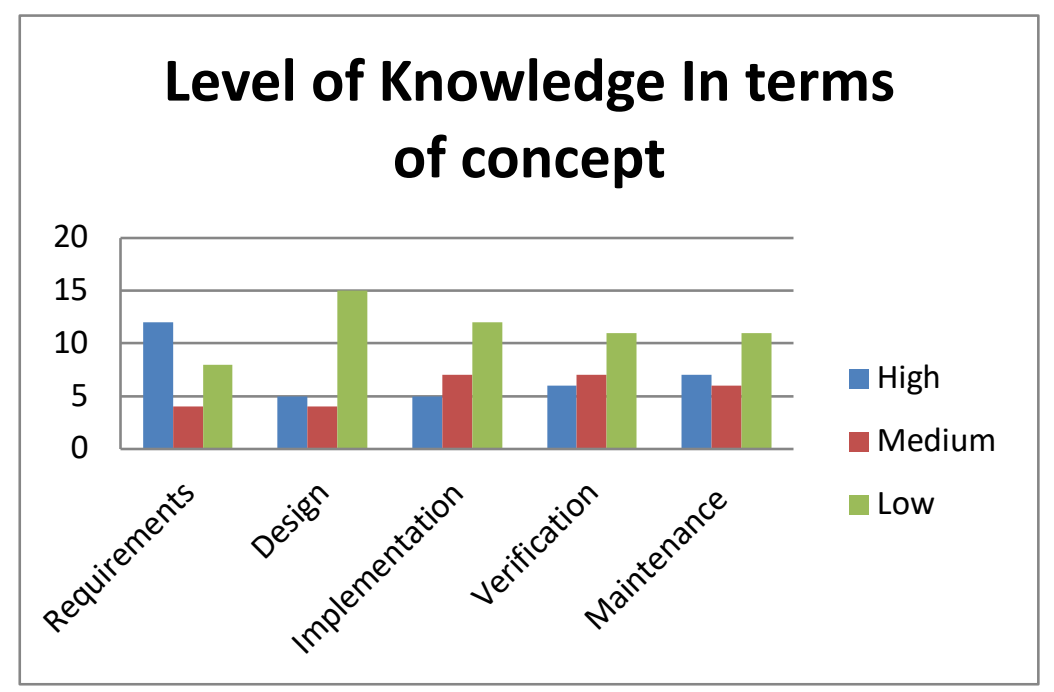

Fig. 12.Level of knowledge measurement in terms of concept

Personalization package: Based on the estimated knowledge level and identified learning style, the personalized learning package is generated for every student (learner). For example, Learner99 belongs to participatory social grouping. In this group, learners prefer learning objects which are in in the forms of image, video, animation, hypertext and chat application. Since Learner99 is having low knowledge level in implementation, the learning package will be provided for him/her in the form of the above-mentioned type/s related to implementation (see Figure 13). This package has been generated using the adaptation algorithm discussed previously in section 4. Using the same method, the personalized learning packages are generated for the other participants. 


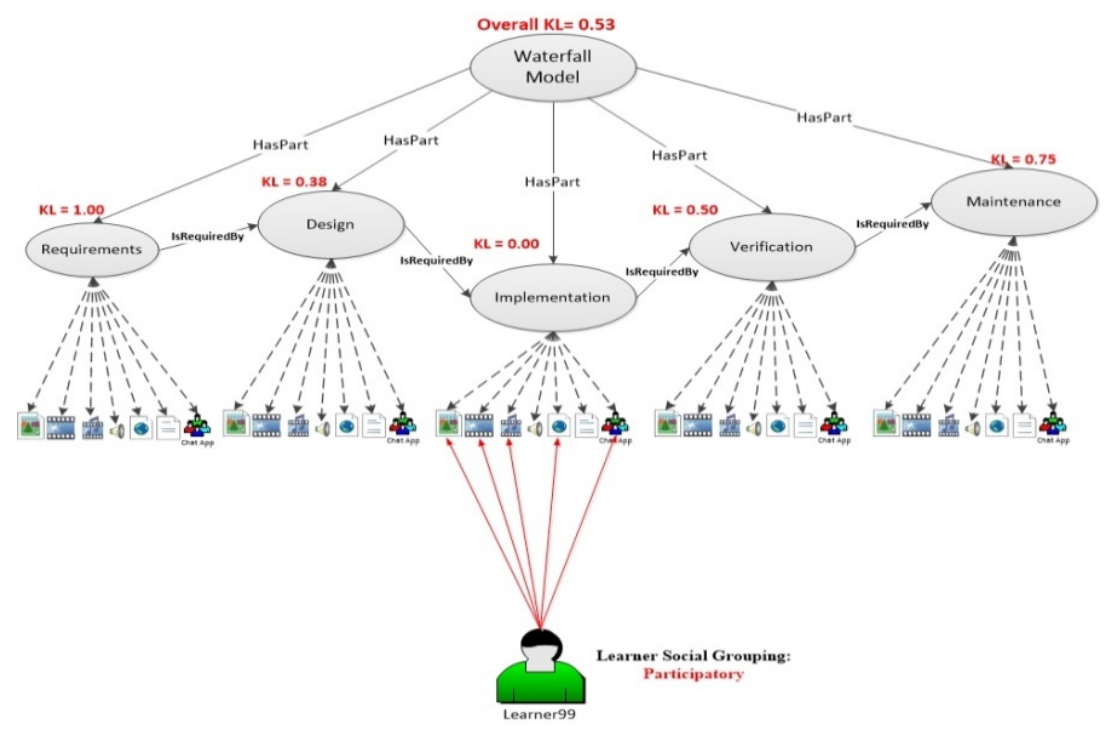

Fig. 13.Personalized learning package for Learner99

The overall indication of the experiment is satisfactory. The adoption of the proposed model enables us to reach the identification of learning style and the estimation of knowledge level.

\section{Conclusion and Future Work}

With the growth of interest in using online collaboration tools for learning activities and discussion, utilizing the generated chat conversations is required for providing personalized e-learning environment. These conversations contain valuable information related to the learner's characteristics and the domain under discussion. Therefore, in order to extract the explicit and implicit valuable information, this paper proposed a mining chat conversation model for information extraction. The paper focused on extracting the semantic relations between the components of a predefined domain ontology. The domain ontology represents the learning activity task with the related term of concepts and learning objects explaining these terms. Using a structured collaboration tool and natural language processing techniques, the collected data has been categorized and analyzed. The analysis of the experiment targeted the structuring of the domain ontology and the identification of two learner's characteristics (knowledge level and learning style). The identified characteristics are the parameters to provide a personalized package consists of specific types of learning objects for a particular term of concept based on the learning style. The proposal has been tested on a small group of learners with acceptable results. However, to promote the proposed model, there is a need to evaluate it using a larger group or more courses in the computer science field. This task is intended to be future work. 


\section{Acknowledgements}

This work is supported by Ministry of Manpower, Sultanate of Oman.

\section{References}

[1] A. Al-Abri et al., "Identifying Learning Styles from Chat Conversation using OntologyBased Dynamic Bayesian Network Model," in 2018 8th International Conference on Computer Science and Information Technology (CSIT), 2018, pp. 1-8. https://doi.org/10.1109/ csit.2018.8486169

[2] A. Al-Abri, Z. Al-Khanjari, N. Kraiem, and Y. Jamoussi, "A scheme for extracting information from collaborative social interaction tools for personalized educational environments," in Proceedings of the IEEE International Conference on Computing, Networking and Informatics, ICCNI 2017, 2017, vol. 2017-Janua. https://doi.org/10.1109/iccni.2017. $\underline{8123795}$

[3] G. Zayaraz, "Concept relation extraction using Naïve Bayes classifier for ontology-based question answering systems," J. King Saud Univ. Inf. Sci., vol. 27, no. 1, pp. 13-24, 2015. https://doi.org/10.1016/j.jksuci.2014.03.001

[4] M. G. H. Al Zamil and Q. Al-Radaideh, "Automatic extraction of ontological relations from Arabic text,” J. King Saud Univ. Inf. Sci., vol. 26, no. 4, pp. 462-472, 2014. https:// doi.org/10.1016/j.jksuci.2014.06.007

[5] S. Pandit, "Ontology-guided extraction of structured information from unstructured text: Identifying and capturing complex relationships," 2010. https://doi.org/10.31274/etd$\underline{180810-2519}$

[6] K. Bontcheva and L. Derczynski, "Extracting Information from Social Media with GATE," 2016. https://doi.org/10.1016/b978-1-84334-749-1.00006-8

[7] J. Cowie and Y. Wilks, "Handbook of natural language processing. chapter Information Extraction." Marcel Dekker, New York, 2000.

[8] R. Y. K. Lau, D. Song, Y. Li, T. C. H. Cheung, and J.-X. Hao, "Toward a fuzzy domain ontology extraction method for adaptive e-learning," IEEE Trans. Knowl. Data Eng., vol. 21, no. 6, pp. 800-813, 2009. https://doi.org/10.1109/tkde.2008.137

[9] M. Šimko, "Automated Acquisition of Domain Model for Adaptive Collaborative WebBased Learning.," Inf. Sci. Technol. Bull. ACM Slovakia, vol. 4, no. 2, pp. 1-9, 2012.

[10] M. Simko and M. Bielikova, "Lightweight domain modeling for adaptive web-based educational system,” J. Intell. Inf. Syst., vol. 52, no. 1, pp. 165-190, 2019. https://doi.org/10. 1007/s10844-018-0518-3

[11] Y. Jamoussi, Z. A. Al-khanjari, and N. Kraiem, "A Framework to Evaluate E-learning Based on Social Networking," vol. 2, no. 4, pp. 26-42, 2014.

[12] Ö. Özyurt and C. Köse, "Chat mining: Automatically determination of chat conversations' topic in Turkish text based chat mediums," Expert Syst. Appl., vol. 37, no. 12, pp. 87058710, 2010. https://doi.org/10.1016/j.eswa.2010.06.053

[13] Y. Jamoussi and A. Y. Nouira, "An extracting model for constructing actions with improved part-of-speech tagging from social networking texts," in Proceedings of 2017 11th International Conference on Intelligent Systems and Control, ISCO 2017, 2017, pp. 77-81. https://doi.org/10.1109/isco.2017.7855957

[14] B. Batrinca and P. C. Treleaven, "Social media analytics: a survey of techniques, tools and platforms," AI Soc., vol. 30, no. 1, pp. 89-116, 2014. https://doi.org/10.1007/s00146-014$\underline{0549-4}$ 
[15] M. Hanini, “Text Modeling in Adaptive Educational Chat Room,” vol. 103, no. 5, pp. 3337, 2014. https://doi.org/10.5120/18073-9022

[16] M. Al-Yahya, R. George, and A. Alfaries, "Ontologies in E-Learning: Review of the literature,” Int. J. Softw. Eng. its Appl., vol. 9, no. 2, pp. 67-84, 2015.

[17] J. Khan and M. Hardas, "A technique for representing course knowledge using ontologies and assessing test problems," in Advances in Intelligent Web Mastering, Springer, 2007, pp. 174-179. https://doi.org/10.1007/978-3-540-72575-6_28

[18] R. Neches et al., "Enabling Technology for Knowledge Sharing," Ai Mag., vol. 12, no. 3, pp. 36-56, 1991.

[19] S. Trausan-Matu and T. Rebedea, "Ontology-based analyze of chat conversations. An urban development case," in Proceedings of Towntology Conference, Liege, 2009.

[20] A. Auger and C. Barrière, "Pattern-based approaches to semantic relation extraction: A state-of-the-art," Terminol. Int. J. Theor. Appl. Issues Spec. Commun., vol. 14, no. 1, pp. $1-19,2008$.

[21] F. M. Khan, T. A. Fisher, L. Shuler, T. Wu, and W. M. Pottenger, "Mining chat-room conversations for social and semantic interactions," Comput. Sci. Eng. Lehigh Univ., 2002.

[22] C. Romero, M. I. López, J. M. Luna, and S. Ventura, "Predicting students' final performance from participation in on-line discussion forums," Comput. Educ., vol. 68, no. October, pp. 458-472, 2013. https://doi.org/10.1016/j.compedu.2013.06.009

[23] J.-S. Wong, B. Pursel, A. Divinsky, and B. J. Jansen, "Analyzing MOOC discussion forum messages to identify cognitive learning information exchanges," in Proceedings of the 78th ASIS\&T Annual Meeting: Information Science with Impact: Research in and for the Community, 2015, p. 23. https://doi.org/10.1002/pra2.2015.145052010023

[24] M. Sankat, R. S, and S. Jaloree, "A Framework for Building Ontology in Education Domain for Knowledge Representation," vol. 14, no. 3, p. 3154033, 2016.

[25] M. H. Ymoun, "CSCL Chats Analysis and Scientific Threads Discovery," in 2018 8th International Conference on Computer Science and Information Technology (CSIT), 2018, pp. 62-65. https://doi.org/10.1109/csit.2018.8486197

[26] A. Al-Abri, Y. Jamoussi, Z. AlKhanjari, and N. Kraiem, "Aggregation and Mapping of Social Media Attribute Names Extracted from Chat Conversation for Personalized ELearning," in 2019 4th MEC International Conference on Big Data and Smart City (ICBDSC), 2019, pp. 1-9. https://doi.org/10.1109/icbdsc.2019.8645567

[27] O. Owoputi, B. O’Connor, C. Dyer, K. Gimpel, N. Schneider, and N. a Smith, "Improved Part-of-Speech Tagging for Online Conversational Text with Word Clusters," Proc. NAACL-HLT 2013, no. June, pp. 380-390, 2013.

[28] A. Ritter, S. Clark, Mausam, and O. Etzioni, "Named Entity Recognition in Tweets: An Experimental Study," Proc. 2011 Conf. Empir. Methods Nat. Lang. Process., pp. 1524 1534, 2011.

[29] K. Bontcheva, L. Derczynski, A. Funk, M. a Greenwood, D. Maynard, and N. Aswani, "TwitIE : An Open-Source Information Extraction Pipeline for Microblog Text," Proc. Recent Adv. Nat. Lang. Process., no. September, pp. 83-90, 2013.

[30] K. Gimpel et al., "Part-of-Speech Tagging for Twitter: Annotation, Features, and Experiments," Proc. 49th Annu. Meet. Assoc. Comput. Linguist. Shortpapers, no. 2, pp. 42-47, 2011.

[31] T. Wang, Y. Li, K. Bontcheva, H. Cunningham, and J. Wang, "Automatic extraction of hierarchical relations from text," in European Semantic Web Conference, 2006, pp. 215229. https://doi.org/10.1007/11762256 18 
[32] B. S. El Zraie, "Extraction of Taxonomic Relations from Arabic Text for Ontology Con-

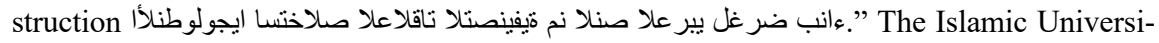
ty-Gaza, 2016.

[33] A. Schutz and P. Buitelaar, "Relext: A tool for relation extraction from text in ontology extension," in International semantic web conference, 2005, pp. 593-606. https://doi.org/10. $\underline{1007 / 11574620 \quad 43}$

[34] I. Kallel and H. C. Fourati, "Integrating Emotion Extraction from Text into Moodle Elearning Platform," in 2018 17th International Conference on Information Technology Based Higher Education and Training (ITHET), 2018, pp. 1-8. https://doi.org/10.1109/ith et.2018.8424795

[35] R. Felder and L. Silverman, "Learning and teaching styles in engineering education," Eng. Educ., vol. 78, no. June, pp. 674-681, 1988.

[36] V. Balakrishnan and C. L. Gan, "Students' learning styles and their effects on the use of social media technology for learning," Telemat. Informatics, vol. 33, no. 3, pp. 808-821, 2016.

[37] A. Laks, "Focusing On Learners," Epsilon Learning Systems, 2017. [Online]. Available: http://epsilonlearning.com/learners.html. [Accessed: 21-Nov-2017]. https://doi.org/10.10 $16 /$ j.tele.2015.12.004

[38] W. C. Paredes and K. S. K. Chung, "Modelling learning \& performance," Proc. 2nd Int. Conf. Learn. Anal. Knowl. - LAK'12, no. May, p. 34, 2012.

\section{Authors}

Amal Bati Said Al-Abri is currently a $\mathrm{PhD}$ candidate in the Department of Computer Science, College of Science at Sultan Qaboos University, Sultanate of Oman. She received her MSc in information technology from Loughborough University, UK. She worked as a lecturer for more than 9 years at Ibri college of Technology in Oman.

Zuhoor Abdullah Salim Al-Khanjari is a professor in software engineering. She worked as the HOD of the Department of Computer Science, College of Science at Sultan Qaboos University, Sultanate of Oman. She received her BSc in mathematics and computing from Sultan Qaboos University, Sultanate of Oman, $\mathrm{MSc}$ and $\mathrm{PhD}$ in computer science (software engineering) from the University of Liverpool, UK. Her research interests include software engineering, software testing techniques, database management, e-learning, mlearning and mobile computing. Currently, she is the coordinator of the software engineering group in the Department of Computer Science, Sultan Qaboos University, Sultanate of Oman. Also, she is coordinating elearning facilities in the same department. She is a member in the editorial board of the International Arab Journal of Information Technology (IAJIT) and a member in the executive committee of the International Arab Conference on Information Technology (ACIT).

Yassine Jamoussi is a HDR between University of Manouba - University of Toulouse - France). He is currently assistant professor of computer science at Sultan Qaboos University. His research interests include process-centered environment, modeling, and meta-modeling of flexible process, process enactment, modeling variability, adaptation, evaluation of process model, service oriented architecture. His research work has been supported by several funding, such as CMCU (Europeen), INRIA 
(French), MRT (Ministry of Research and Technology and Industry of Tunisia), IG (SQU Oman). He has been invited to present his research in many countries in North America, Europe, Africa and in the Middle East. Dr Yassine Jamoussi has been conference chair of IEEE conferences and member of over 20 program committees. He published 20 Journal and 40 conference papers in International journals and conference proceedings.

Naoufel Kraiem is currently an associate professor of computer science at SQU. His research interests include IT adoption and usage information modelling, software engineering, software product lines and CASE tools. His research work has been supported by funding of the CNRS, INRIA, MRT (Ministry of Research and Technology and Industry) and by the Commission of the European Communities under the ESPRIT Programmes (BUSINESS CLASS). He has had several articles published in many journals such as a management science information systems research communications of the ACM and IEEE Transactions. He has been invited to present his research in many countries in North America, Europe, Africa and in the Middle East. Pr Naoufel Kraiem has been a member of over 20program committees.

Article submitted 2019-06-14. Resubmitted 2019-08-10. Final acceptance 2019-08-12. Final version published as submitted by the authors. 